\title{
CHARACTERIZATION OF PROTOTYPE LSST CCDs
}

\author{
P. O'Connor ${ }^{\mathrm{a}}$, J. Frank $\mathrm{k}^{\mathrm{a}}$, J.C. Geary ${ }^{\mathrm{b}}$, D.K. Gilmore ${ }^{\mathrm{c}}$, \\ I. Kotov ${ }^{\text {a }}$, V. Radeka ${ }^{\mathrm{a}}$, P. Takacs ${ }^{\mathrm{a}}$, and J.A. Tyson ${ }^{\mathrm{d}}$$$
* * *
$$ \\ ${ }^{a}$ Brookhaven National Laboratory \\ Upton, NY 11973-5000 \\ ${ }^{\mathrm{b}}$ Harvard-Smithsonian Center for Astrophysics \\ Cambridge, MA 02138 \\ 'Stanford Linear Accelerator Center \\ Menlo Park, CA \\ ${ }^{\mathrm{d}}$ University of California at Davis \\ Davis, CA 95616
}

June, 2008

*This manuscript has been authored by Brookhaven Science Associates, LLC under Contract No. DC-AC02-98CH10886 with the U.S. Department of Energy. The United States Government retains, and the publisher, by accepting the article for publication, acknowledges, a world-wide license to publish or reproduce the published form of this manuscript, or allow others to do so, for the United States Government purposes. 


\section{DISCLAIMER}

This work was prepared as an account of work sponsored by an agency of the United States Government. Neither the United States Government nor any agency thereof, nor any of their employees, nor any of their contractors, subcontractors, or their employees, makes any warranty, express or implied, or assumes any legal liability or responsibility for the accuracy, completeness, or any third party's use or the results of such use of any information, apparatus, product, or process disclosed, or represents that its use would not infringe privately owned rights. Reference herein to any specific commercial product, process, or service by trade name, trademark, manufacturer, or otherwise, does not necessarily constitute or imply its endorsement, recommendation, or favoring by the United States Government or any agency thereof or its contractors or subcontractors. The views and opinions of authors expressed herein do not necessarily state or reflect those of the United States Government or any agency thereof. 


\title{
Characterization of prototype LSST CCDs
}

\author{
P. O'Connor ${ }^{a}$, J. Frank ${ }^{a}$, J.C. Geary ${ }^{b}$, D.K. Gilmore ${ }^{c}$, I. Kotov ${ }^{a}$, V. Radeka ${ }^{a}$, P. Takacs ${ }^{a}$, J.A. \\ Tyson $^{\mathrm{d}}$ \\ ${ }^{\mathrm{a} B}$ Brookhaven National Laboratory, Upton, NY USA $11973 ;{ }^{\mathrm{b}}$ Harvard-Smithsonian Center for \\ Astrophysics, 60 Garden St., Cambridge, MA USA 02138; 'Stanford Linear Accelerator Center, \\ Menlo Park, CA USA; ${ }^{d}$ University of California, Davis, CA 95616
}

\begin{abstract}
We present characterization methods and results on a number of new devices produced specifically to address LSST's performance goals, including flatness, QE, PSF, dark current, read noise, CTE, cosmetics, and crosstalk. The results indicate that commercially produced, thick n-channel over-depleted CCDs with excellent red response can achieve tight PSF at moderate applied substrate bias with no evidence of persistent image artifacts. We will also report ongoing studies of mosaic assembly techniques to achieve chip-to-chip co-planarity, high fill factor, and thermal stability..
\end{abstract}

Keywords: CCD, LSST, quantum efficiency, point spread function

\section{INTRODUCTION}

The ambitious science goals of the Large Synoptic Survey Telescope (LSST) will be achieved in part by a wide-field imager that will achieve a new level of performance in terms of area, speed, and sensitivity. The instrument performance is dominated by the focal plane sensors, which are now in development. These new-generation sensors will make use of advanced semiconductor technology and will be complemented by a highly integrated electronics package located inside the cryostat. A test laboratory has been set up at Brookhaven National Laboratory (BNL) to characterize prototype sensors and to develop test and assembly techniques for eventual integration of production sensors and electronics into modules that will form the final focal plane.

As described in [1], the key requirements for LSST sensors are wideband quantum efficiency (QE) extending beyond $1 \mu \mathrm{m}$ in the red, control of point spread function (PSF), and fast readout using multiple amplifiers per chip operated in parallel. In addition, LSST's fast optical system ( $f / 1.25)$ places severe constraints on focal plane flatness. At the chip level this involves packaging techniques to minimize warpage of the silicon die, and at the mosaic level careful assembly and metrology to achieve a high coplanarity of the sensor tiles.

In view of the long lead time to develop the needed sensor technology, LSST undertook a study program with several vendors to fabricate and test devices which address the most critical performance features [2]. The remainder of this paper presents key results of this study program. Section 2 summarizes the sensor requirements and the results of design optimization studies, and Section 3 presents the sensor development plan. In Section 4 we describe the test bench at BNL. Section 5 reports measurement results obtained to date on devices fabricated by several vendors. Section 6 presents a summary of the paper and an outlook for the future work.

This manuscript has been co-authored by employees of Brookhaven Science Associates, LLC. Portions of this work are supported by the Department of Energy under contract DE-AC02-76SF00515 with the Stanford Linear Accelerator Center, contract DE-AC0298CH10886 with Brookhaven National Laboratory, and contract W-7405-ENG-48 with Lawrence Livermore National Laboratory. Additional funding comes from private donations, grants to universities, and in-kind support at Department of Energy laboratories and other LSSTC Institutional Members..

"poc(a),bnl.gov; phone 1631 344-7577; fax $1631344-5773$ 


\section{SENSOR REQUIREMENTS AND DESIGN OPTIMIZATION}

\subsection{Requirements}

The technology advances needed for LSST sensors are listed in Table 1 below.

Table 1 LSST sensor requirements

\begin{tabular}{lll}
\hline \multicolumn{1}{c}{ Science driver } & \multicolumn{1}{c}{ Technology Advance } & \multicolumn{1}{c}{ Criterion } \\
\hline Broadband, high QE & Thick silicon, fully depleted & $\mathrm{QE}(1000 \mathrm{~nm})>30 \%$ \\
& Transparent back contact & $\mathrm{QE}(400 \mathrm{~nm})>40 \%$ \\
Seeing-limited image quality & Low charge diffusion & $<3.2 \mu \mathrm{m} \mathrm{rms}$ \\
& Small pixel size & $10 \mu \mathrm{m}(0.2 ")$ \\
& Low read noise & $<5 \mathrm{e}-\mathrm{rms}$ \\
& Low dark current & $<2 \mathrm{e}-/ \mathrm{pix} / \mathrm{s}$ \\
& Low persistence & $<10^{-4}$ \\
& High full well & $>90,000 \mathrm{e}-$ \\
& Flat silicon surface & $<5 \mu \mathrm{m} \mathrm{p}-\mathrm{v}$ \\
& TTP-controlled package & $<6.5 \mu \mathrm{m}$ over raft \\
& Multiport output & $(4 \mathrm{~K})^{2}, 16$ output \\
High throughput & High fill factor die \& pkg & $>93 \%$ \\
\hline
\end{tabular}

\subsection{Optical}

To balance the tradeoffs between red QE and charge diffusion, an optimization study was performed [1]. Increasing the silicon thickness improves the QE beyond $950 \mathrm{~nm}$, while it causes increased charge diffusion which broadens the PSF in the $u, g, r$, and I bands. An illustration of this tradeoff is shown in Figure 2, where the contribution to PSF at 550nm from charge diffusion is plotted versus averaged QE in the y-band. Note that temperature plays a role in this tradeoff - higher temperatures improve the red $\mathrm{QE}$ while having minimal effect on diffusion.

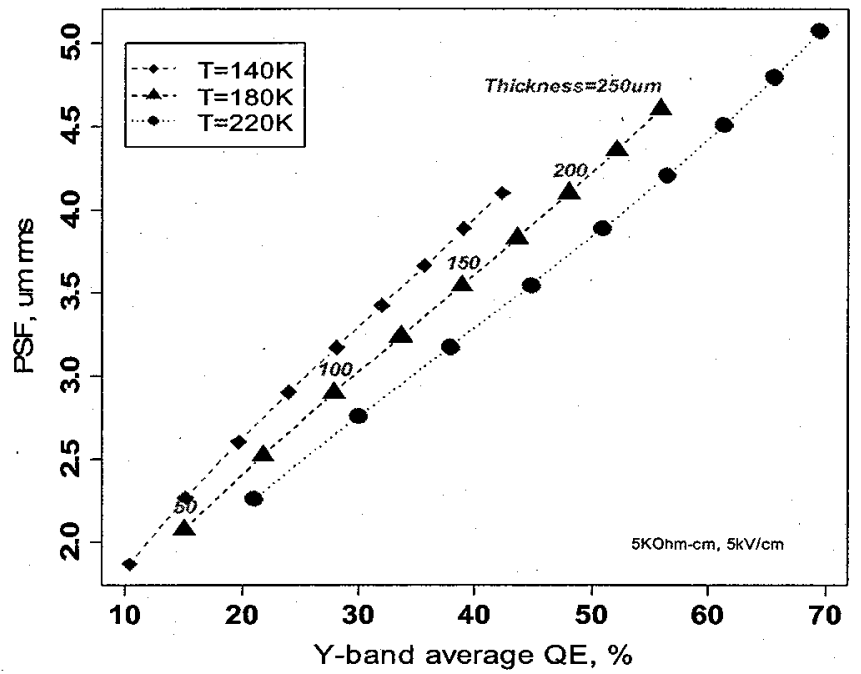

Figure 1 Effect of sensor thickness on point spread function and y-band QE at three temperatures: 140K (diamonds), 180K (triangles), and $220 \mathrm{~K}$ (circles). Thickness range is $50 \mu \mathrm{m}$ (points at lower left) to $250 \mu \mathrm{m}$ (upper right) in $50 \mu \mathrm{m}$ steps.

\subsection{Electrical}

Another tradeoff involves the electrical performance of the focal plane. Survey speed is decreased by the time taken to read out the focal plane after each exposure, but increasing the pixel rate degrades read noise. To meet the goal of $2 \mathrm{~s}$ readout time for the full focal plane while keeping the read noise below 5 electrons rmis, the sensor is divided into segments read out in parallel. In the tradeoff analysis the detector size is assumed to be $4 \mathrm{~K} X 4 \mathrm{~K}$, the readout time is 
constrained to be $2 \mathrm{~s}$, and we estimate the noise performance of the detector based on a simple noise model of the source follower transistor (Figure 2a). The choice of 16 segments (1Mpix per segment) and 250kpix/s provides the necessary performance. The arrangement of the segments can be chosen to provide a large contiguous imaging area, as.shown in the CCD conceptual layout in Figure 2b.
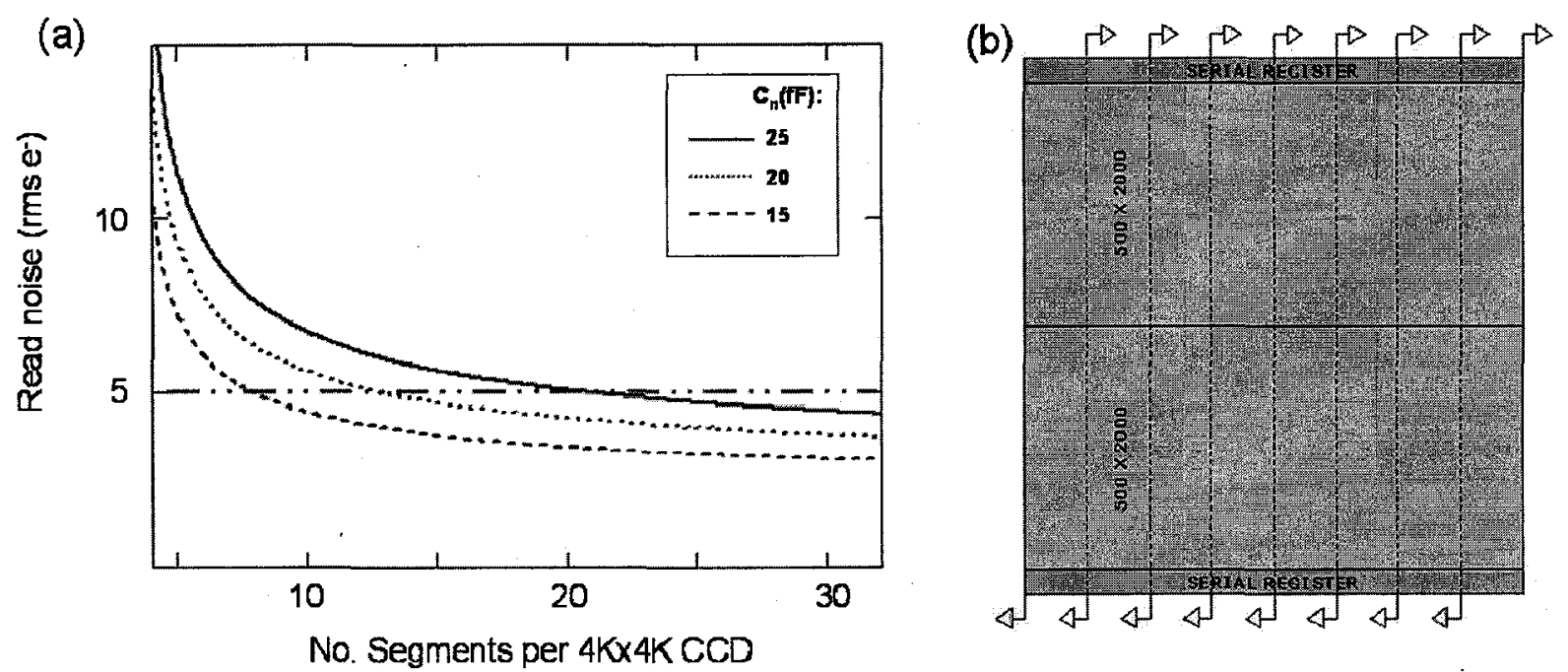

Figure 2: (a)Estimated read noise for a $4 \mathrm{~K}$ x $4 \mathrm{~K}$ sensor constrained to $2 \mathrm{~s}$ readout time. Increasing the segmentation allows the clock rate, and hence the read noise, to be reduced. Noise estimates are based on CCD output transistor properties with three values of sense node capacitance. (b) Proposed layout of a 16 -fold segmented, $4 \mathrm{~K} \times 4 \mathrm{~K} \mathrm{CCD}$ for fast, lownoise readout.

\subsection{Mechanical}

A crude defocus model provides limits on the flatness tolerance. Figure 3 shows the normalized PSF as a function of displacement from best focus. The proposed allocation of flatness tolerance for the sensor surface, raft of nine sensors, and full focal plane of 189 sensors is shown.

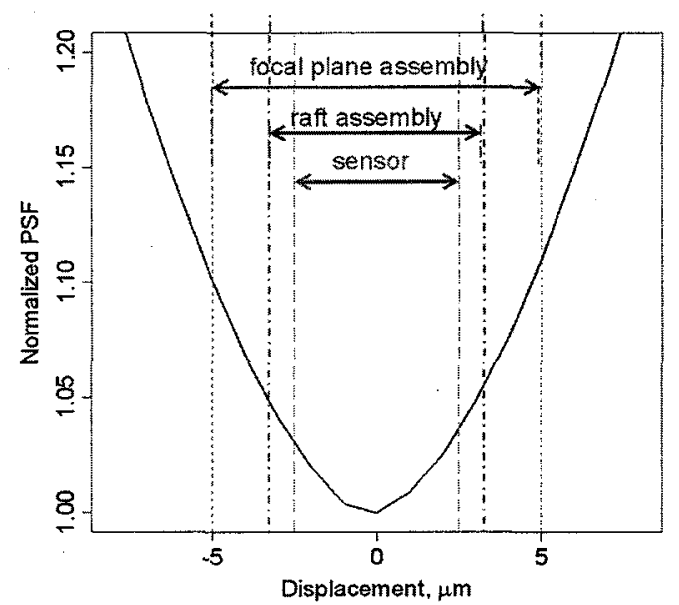

Figure 3: Estimated defocus for 550nm light, with allocation to sensor, raft, and full focal plane. A $3.2 \mu \mathrm{m}$ rms Gaussian PSF at optimum focus is assumed. 


\subsection{Reference design}

A reference design for LSST sensors has been proposed based on the preceding design studies. As shown in Figure 4, it is a $4 \mathrm{~K} \times 4 \mathrm{~K} C C D$ fabricated on $100 \mu \mathrm{m}$-thick, p-type silicon with a resistivity in the range $5-10 \mathrm{k} \Omega-\mathrm{cm}$. The CCD imaging area is divided into sixteen, $512 \times 2048$ pixel, independently read out segments. Each segment has a low power, low noise source follower amplifier. An independent substrate bias voltage, applied from the front side of the device, biases the thin conductive window on the backside and results in a fully-depleted substrate under the imaging area with an average electric field of $5-10 \mathrm{kV} / \mathrm{cm}$. Guard rings around the periphery provide a gradual lateral voltage gradient to the silicon die edge, which is at the same potential as the entrance window. Care must be taken to ensure that pimplanted regions on the frontside are narrow enough to avoid forming conductive channels to the backside. Bond pads are arranged on two sides of the die and connect to traces on the package, which may incorporate bussing of common clock and bias signals.

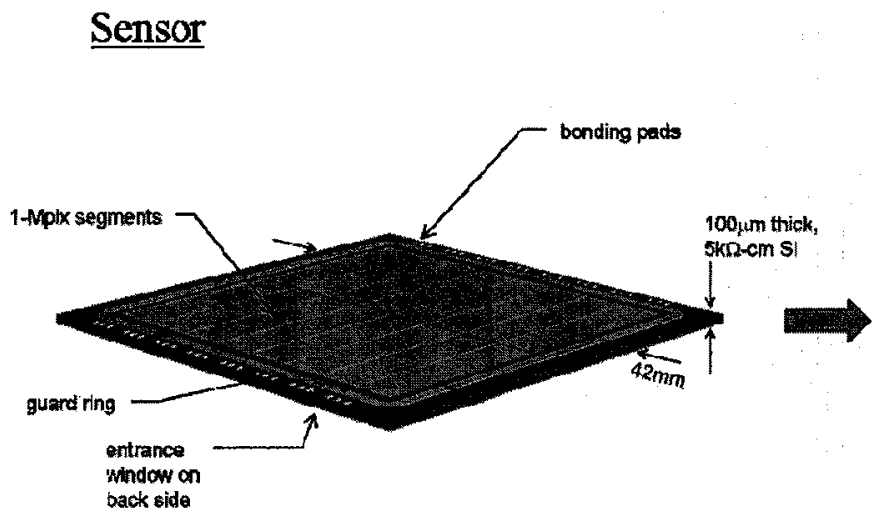

\section{Raft of 9 sensors}
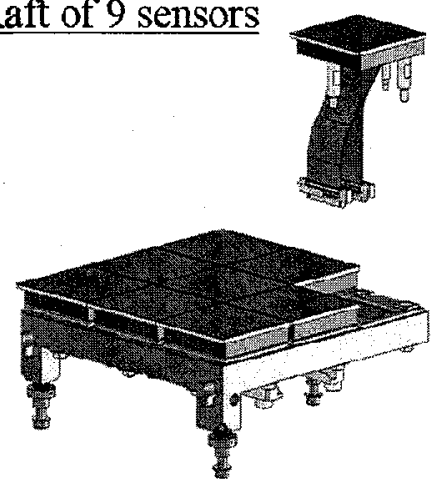

Figure 4: Sensor and raft reference designs

\section{SENSOR DEVELOPMENT PLAN}

The LSST camera collaboration is about halfway through a three-phase sensor technology development program. Starting in 2005, contracts were awarded to three vendors to address the most pressing technology challenges [2]. A matrix showing the goals of the study contracts is shown in Table 2. With the funding available, this program emphasized the demonstration of thick, high resistivity, fully-depleted sensors. Less emphasis was given to high fill factor die layout, packaging, and flatness.

Table 2: Vendor study program.

\begin{tabular}{|l|l|c|c|c|c|c|c|c|}
\hline Vendor & Sensor & $\begin{array}{c}\text { Thick } \\
\text { hi-rho }\end{array}$ & $\begin{array}{c}10 \mu \mathrm{m} \\
\text { pixel }\end{array}$ & $\begin{array}{c}\text { Large } \\
\text { format }\end{array}$ & Segmented & $\begin{array}{c}\text { Flat } \\
\text { Silicon }\end{array}$ & $\begin{array}{c}\text { Buttable } \\
\text { Pkg }\end{array}$ & $\begin{array}{c}\text { Sensors } \\
\text { delivered }\end{array}$ \\
\hline $\mathrm{A}$ & $\mathrm{CCD}$ & $\mathrm{X}$ & - & - & - & - & - & 5 \\
\hline $\mathrm{B}$ & $\mathrm{CCD}$ & $\mathrm{X}$ & $\mathrm{X}$ & $\mathrm{X}$ & $\mathrm{x}$ & - & - & 2 \\
\hline $\mathrm{C}$ & $\mathrm{pin} / \mathrm{CMOS}$ & $\mathrm{X}$ & $\mathrm{X}$ & $\mathrm{X}$ & $\mathrm{X}$ & - & - & 1 \\
\hline
\end{tabular}

Vendors $A$ and $B$ developed CCDs while Vendor $C$ provided a large-format silicon $p-i-n / C M O S$ hybrid. The $p-i-$ $\mathrm{n} / \mathrm{CMOS}$ hybrid sensor has been tested and results are reported in [3]. The interest in $\mathrm{p}-\mathrm{i}-\mathrm{n} / \mathrm{CMOS}$ technology for LSST is now being focused on guide sensors, where fast window-mode readout is advantageous. The remainder of this paper will report the initial results of tests on the study program's CCD sensors.

Following the study program, a request for proposals for prototype sensors meeting all LSST specifications has been issued in early 2008. In addition to meeting performance goals, the prototype phase will emphasize yield and 
reproducibility to minimize risk of cost and schedule overruns in procurement. During prototype development the LSST collaborating institutions will prepare test facilities having throughput compatible with the expected production rate of $10-20$ sensors per month. Development of the production-ready prototype is expected to take two years, after which procurement of the full complement of science sensors will begin.

\section{TEST FACILITY}

A CCD test facility has been constructed in the Instrumentation Division of Brookhaven National Laboratory. Its primary instrumentation consists of:

- Liquid nitrogen cooled Dewar with 4" window, temperature controlled stage for mounting CCDs in a variety of mechanical packages. A $10 \mu \mathrm{Ci}{ }^{55} \mathrm{Fe}$ source, in the form of a $3 \mathrm{~mm}$ disk, is mounted on a stepper-motor actuated arm inside the Dewar which can position the source over the center of the CCD or behind a shield.

- Monochromatic flat field illumination provided by a Xe lamp, grating monochromator, and 12 " integrating sphere with input, monitor, and output ports.

- Long working distance, high N.A. microscope objective mounted on a translation stage, which images a $4 \mu \mathrm{m}-$ core optical fiber onto the CCD [4]. For $650 \mathrm{~nm}$ light the spot size on the $C C D$ is about $1.2 \mu \mathrm{m}$ Gaussian rms.

- Non-contact flatness measurement system consisting of an optical confocal displacement sensor (Keyence LT9030) mounted on a $200 \mathrm{~mm}$-travel $\mathrm{xy}$ translation stage. The accuracy of the flatness measurement is presently limited by the nonidealities of the stage to about $2 \mu \mathrm{m}$.

All testbench apparatus can be controlled over RS-232 or GPIB.

Our CCD controller is based on the control and readout electronics developed for the MMT Megacam [5]. For our measurements the readout rate was fixed at $156 \mathrm{kpix} / \mathrm{s}$. Communication with the Linux PC is by optical fiber interface [6].

We use the RTS-2 observatory manager software [7] to provide a framework by which the user can specify a sequence of measurement runs, using a flexible scripting language. Using RTS-2, we have developed scripts that perform a complete optical and electrical characterization of the CCD under test. The run set (for measurement at three temperatures) generates about 850 image files and runs unattended for about $6-8$ hours, typically overnight. Automated analysis procedures perform data reduction and various data quality checks.

Quantum efficiency is determined by taking flat field exposures over a range of wavelengths from $300-1100 \mathrm{~nm}$ and subtracting a mean dark frame from each. The $\mathrm{QE}$ is then defined as

$$
Q E(\lambda, T)=\frac{\left\langle S_{A D U}(\lambda, T)>\right.}{g(T)} * F_{r e f}(\lambda) * R(\lambda)
$$

Where $<S_{A D U}>$ is the signal (in ADU per pixel) averaged over a large defect-free area of the CCD, $g(T)$ is the electronic gain in ADU per electron, $F_{r e f}$ is the photon flux measured by the monitor photodiode in the integrating sphere, and $R$ is the predetermined ratio of flux at the CCD location to flux at the monitor photodiode. The factor $g$ is measured by finding the $\mathrm{K}_{\alpha}$ and $\mathrm{K}_{\beta}$ peaks in the spectrum of xray hits from ${ }^{55} \mathrm{Fe}$, or by the slope of the photon transfer curve (variance vs. mean). The ratio $R$ is determined by placing a calibrated photodiode at the CCD location, scanning the monochromator over the wavelength range $300-1100 \mathrm{~nm}$, and recording the ratio of flux in the photodiode at the CCD position to that in the integrating sphere. Reproducibility of the QE measurement is below $1 \%$ in the wavelength range $400-900 \mathrm{~nm}$. Absolute accuracy is limited by the histogram-fitting procedure in determining $g(T)$, and by uncertainty in the temperature dependence of the soft $x$-ray energy required to produce an electron-hole pair in silicon. We do not have a firm estimate of these systematic errors, but believe them to be good to better than $5 \%$.

For PSF measurements, we use the virtual knife edge technique described in [8], [9]. The spot produced by the point projector is repeatedly imaged as it is scanned in $2 \mu \mathrm{m}$ steps in x or y. Integrating the flux in a fixed group of pixels through which the spot travels allows one to estimate the size and shape of the point spread function. We fit the VKE profile to a Gaussian, and subtract the predetermined optical spot size quadratically to get an estimate of the PSF due to charge diffusion effects in the CCD. 
Figure 5 gives examples of an $\mathrm{x}$-ray histogram (a) and a VKE scan (b), with fits to the data.

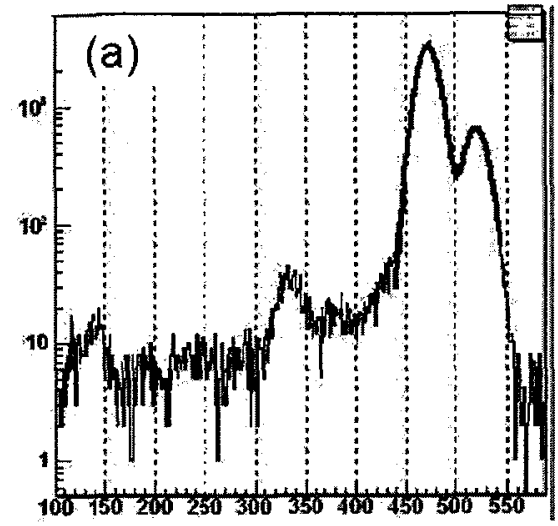

(b)
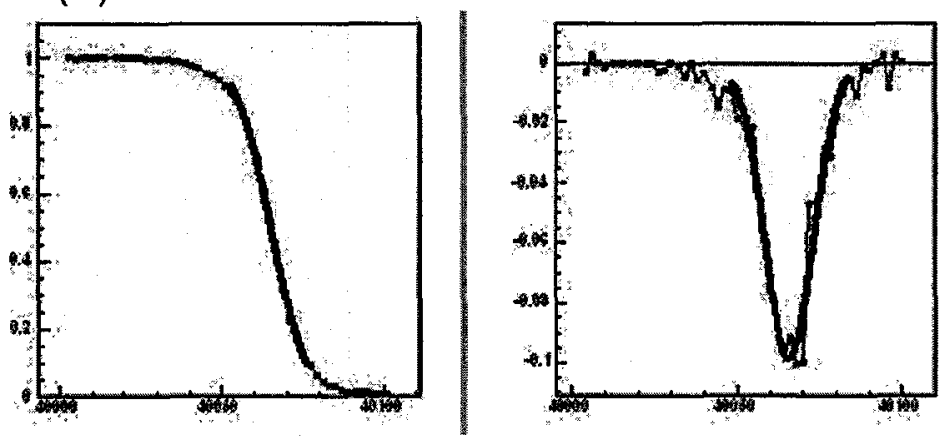

Figure 5: (a) $\mathrm{x}$-ray histogram from ${ }^{55} \mathrm{Fe}$, with fit to $\mathrm{K}_{\alpha}$ and $\mathrm{K}_{\beta}$ peaks at 470 and 520 ADU. (b) Virtual knife edge scan, showing integral and differential fits.

\section{TEST RESULTS ON STUDY CONTRACT CCDS}

\subsection{Description of CCDs delivered}

Five devices from vendor A have been received and tested at BNL. Devices received early in the study program received less rigorous testing than those received later, as test equipment and analysis routines underwent improvement. Vendor $\mathrm{B}$ delivered two devices, but these have not yet been tested at BNL. Both vendors supplied operating parameters and test data for their CCDs. Table 3 is a summary of the characteristics of each device.

\begin{tabular}{|c|c|c|c|c|c|c|c|}
\hline Vendor & Device & Thickness & Pixel size & Format & Segmentation & Amplifier & Comment \\
\hline A & $106-03$ & $100 \mu \mathrm{m}$ & $13.5 \mu \mathrm{m}$ & $2 \mathrm{~K} \times 512$ & 1 or 2 & 1-stage & $\begin{array}{l}\text { "setup" } \\
\text { device }\end{array}$ \\
\hline A & $106-05$ & $100 \mu \mathrm{m}$ & $13.5 \mu \mathrm{m}$ & $2 \mathrm{~K} \times 512$ & 1 or 2 & 1-stage & \\
\hline A & $106-06$ & $100 \mu \mathrm{m}$ & $13.5 \mu \mathrm{m}$ & $2 \mathrm{~K} \times 512$ & 1 or 2 & 2-stage & \\
\hline A & $106-07$ & $100 \mu \mathrm{m}$ & $13.5 \mu \mathrm{m}$ & $2 \mathrm{~K} \times 4.6 \mathrm{~K}$ & 1 or 2 & 2-stage & $\begin{array}{l}\text { Testing } \\
\text { incomplete }\end{array}$ \\
\hline A & $107-01$ & $150 \mu \mathrm{m}$ & $13.5 \mu \mathrm{m}$ & $2 \mathrm{~K} \times 512$ & 1 or 2 & 2-stage & $\begin{array}{l}\text { Different } \\
\text { process lot }\end{array}$ \\
\hline$B$ & $108-01$ & $100 \mu \mathrm{m}$ & $10 \mu \mathrm{m}$ & $4 \mathrm{~K} \times 4 \mathrm{~K}$ & 16 & 1-stage & \\
\hline$B$ & $108-02$ & $100 \mu \mathrm{m}$ & $10 \underline{\mu m}$ & $4 \mathrm{~K} \times 4 \mathrm{~K}$ & 16 & 1 -stage & \\
\hline
\end{tabular}

\subsection{Flatness}

Four of the five devices from Vendor A have been measured for surface flatness, and the results are shown in Figures 6 and 7. The contour plots give the residuals from the best-fit plane to data taken on a $1 \times 1 \mathrm{~mm}$ grid. Below each contour map is a graph showing the cumulative distribution of residuals (absolute values shown). These devices show a characteristic bowing, with the large device being center-high and the smaller devices center-low. The departure from flatness is within LSST specifications over more than $96 \%$ of the imaging area for these devices. One of Vendor B's devices has been measured and it exhibits a poorer flatness characteristic, with an rms deviation from plane about seven 
times larger than the devices from Vendor A. However, neither vendor made any special attempt to produce flat devices for this study program.

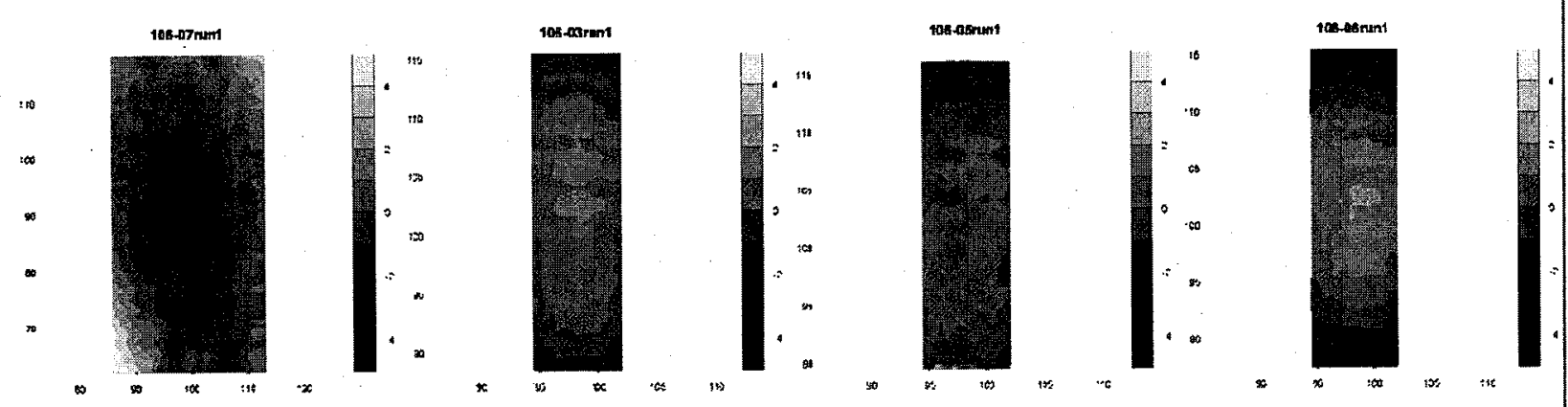

Figure 6: Flatness contours for Vendor A devices. Contour interval is one micron.
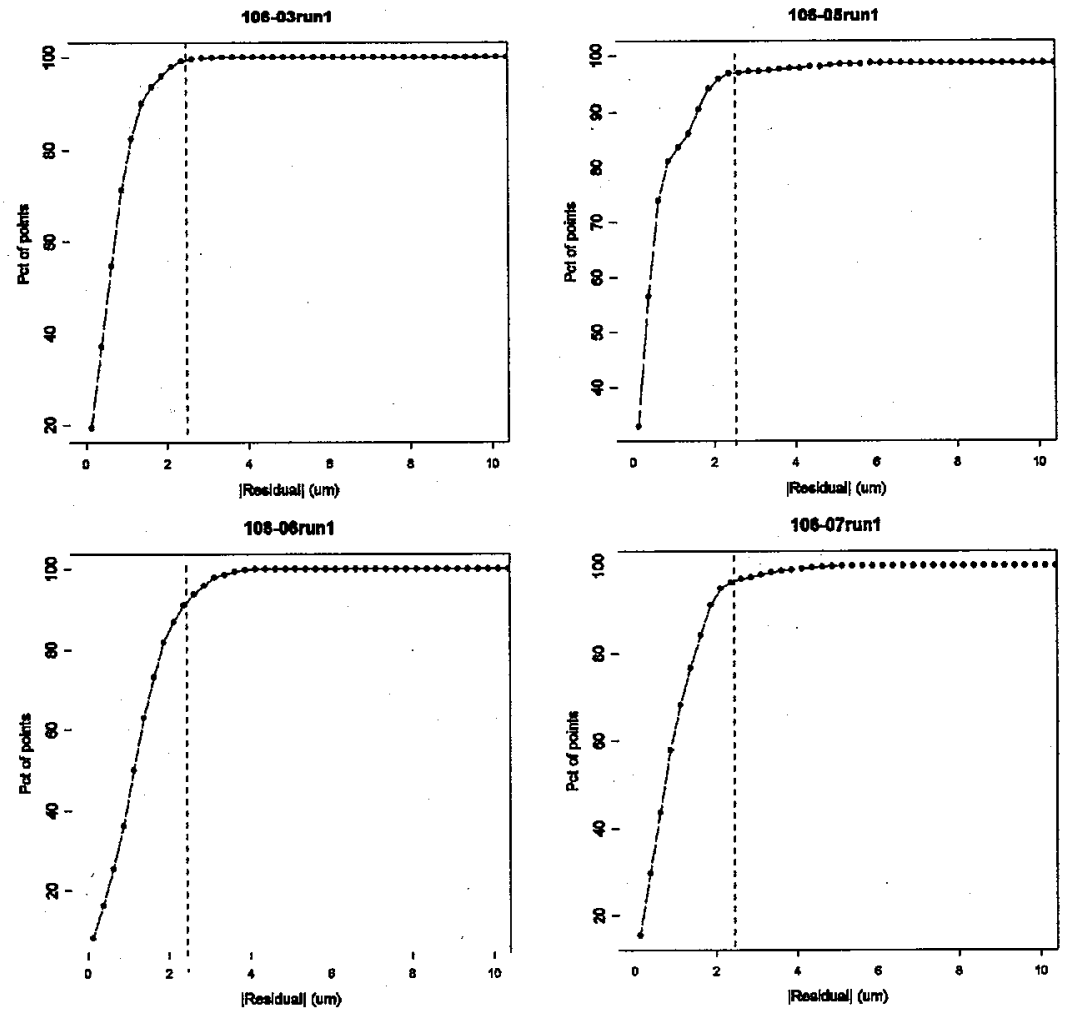

Figure 7: Flatness of Vendor A sensors.Plots on right show cumulative distribution of flatness (absolute value of residuals to best-fit plane). Vertical dashed line is LSST spec.

\subsection{Dark current and defects}

Dark current is measured by taking the slope of the signal-versus-time linear fit for each pixel, in a series of dark runs of 10 to $500 \mathrm{~s}$. Figure 8 shows the mean dark current, and the fraction of pixels with greater than $2 \mathrm{e}-/$ pixel/s dark current, as a function of temperature for device 106-06. Other devices from Vendor A behave similarly. Bright defects, which are prominent at room temperature, are extinguished at lower temperature or with lower substrate bias. 

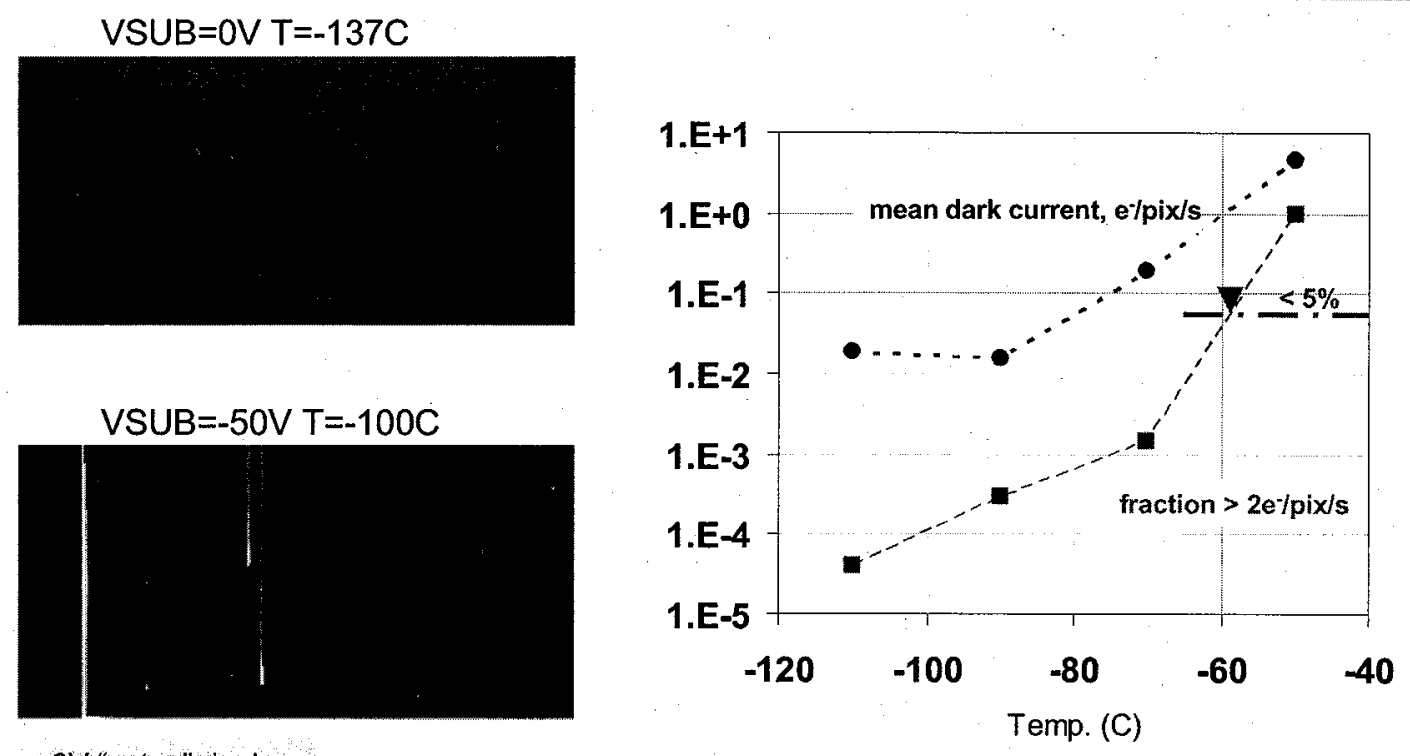

e2V "setup" device

Figure 8: Dark images ("setup" device 106-03) and dark current vs temperature (study contract deliverable device 106-06). Circles: mean dark current. Squares: fraction of pixels with $>2 \mathrm{e}-/ \mathrm{pix} / \mathrm{s}$. LSST spec is for less than $.05 \%$ of pixels to have $2 \mathrm{e}-/ \mathrm{pix} / \mathrm{s}$.

\subsection{Amplifier gain, linearity, and full well}

Figure 9 shows the response of sensor 106-06 to flatfield illumination at increasing exposure times. The full well (Fig. $9(\mathrm{a})$ ) is about 300,000 electrons. Fig. 9 (b) shows that the response is linear to within $0.5 \%$ from zero to $90 \%$ of full well.

(a)

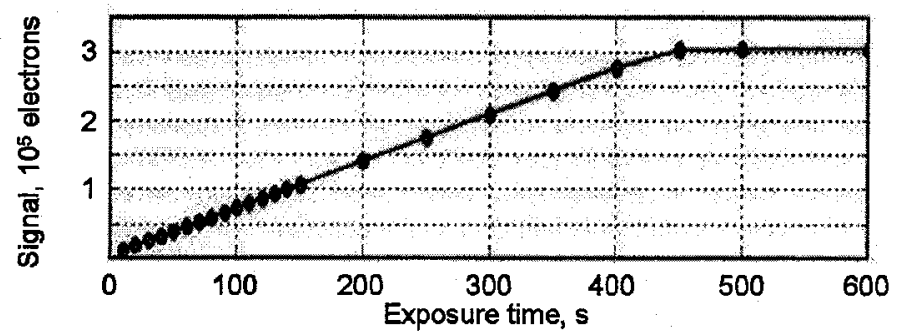

(b)

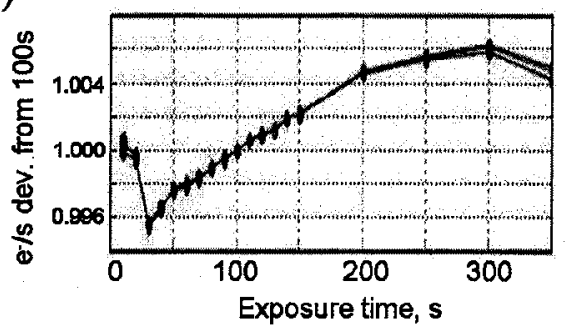

Figure 9 (a) Response to flatfield illumination. (b) Slope normalized to the value at 100 s. Response is linear up to $90 \%$ of full well. 


\subsection{Quantum efficiency}

Quantum efficiency in the range $300-1100 \mathrm{~nm}$ is shown in Figure 10. Data is for devices of $100 \mu \mathrm{m}$ thickness.

Vendor A

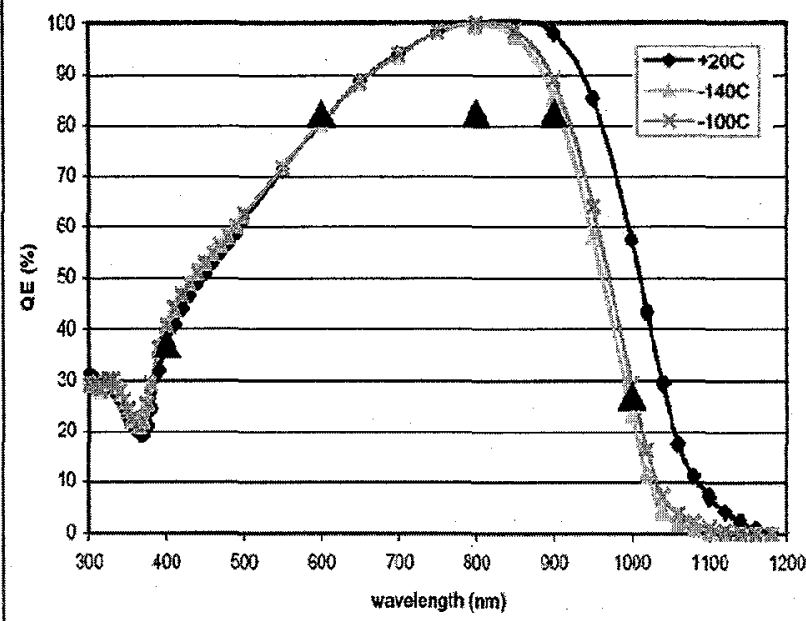

Vendor B

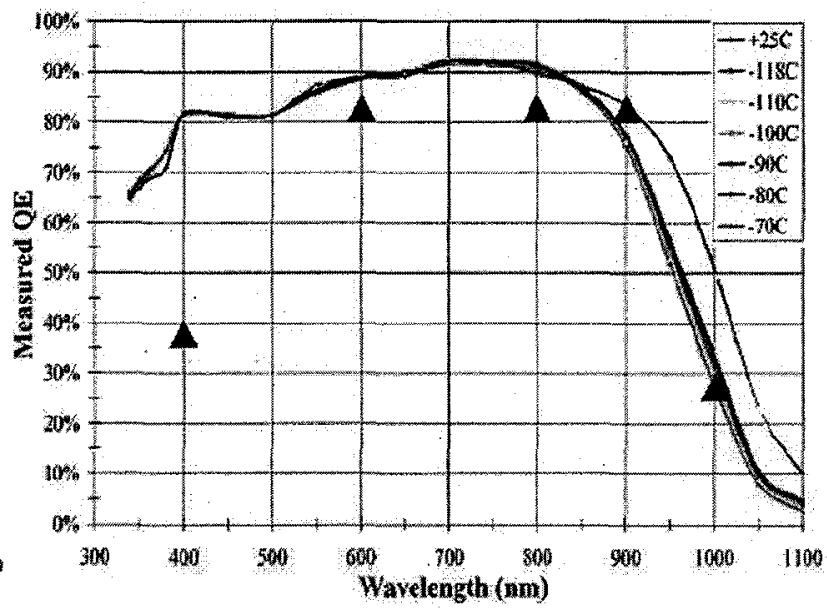

Figure 10. Quantum efficiency for $100 \mu \mathrm{m}$-thick devices biased at full depletion. Triangles are LSST requirements. Measurements supplied by vendors.

In Figure 11 we show the quantum efficiency of 100 and $150 \mu$ m-thick devices from Vendor A. For these devices we have also compared the measured $\mathrm{QE}$ to values predicted from a model that takes into account the absorption coefficient of silicon at $-70 \mathrm{C}$ from [10], the properties of the antireflection coating determined by ellipsometry, and an assumed dead layer of $6 \mathrm{~nm}$ at the entrance window.
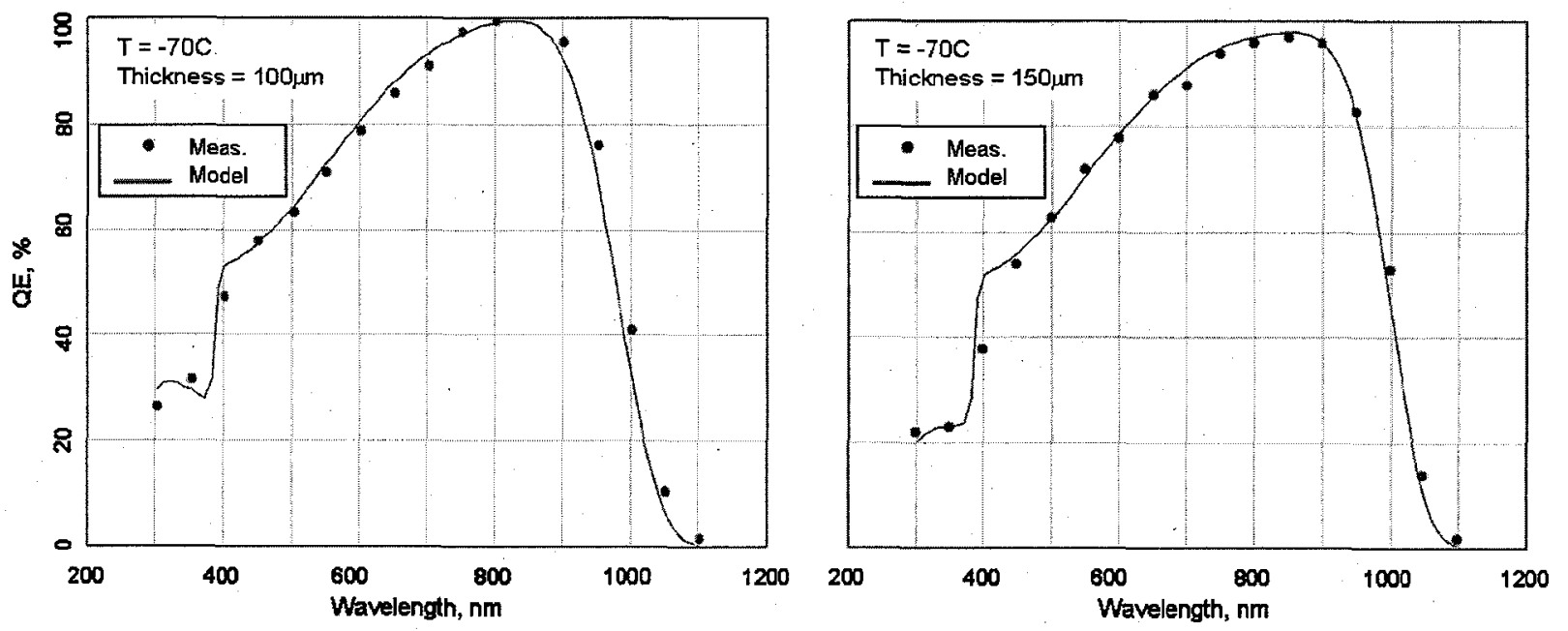

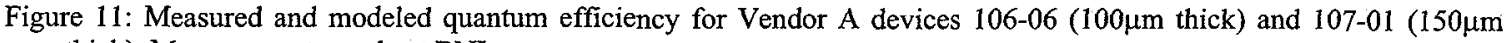
thick). Measurements made at BNL.

\subsection{Point spread function}

The point spread function measured by the virtual knife edge technique is shown in Fig. 12 for two samples from Vendor A. Data for both samples was taken at $163 \mathrm{~K}$. By fitting the low-voltage portion of the curve the doping concentration, silicon thickness, and junction potential can be determined to be $4.1 \times 10^{12} \mathrm{~cm}^{-3,}, 95 \mu \mathrm{m}, 18 \mathrm{~V}$ for sample 106-05 and 
$2.1 \times 10^{12} \mathrm{~cm}^{-3}, 150 \mu \mathrm{m}, 18 \mathrm{~V}$ for sample $107-01$ respectively. As the voltage is made more negative the CCDs become fully depleted and the PSF is determined by the diffusion of electrons as

$$
\frac{\sigma}{d}=\sqrt{2 D_{T} t_{d r}}
$$

where $d$ is the silicon thickness, $D_{T}$ is the transverse diffusion coefficient, and $t_{d r}$ is the drift time. Fig. 12 shows two curves for the fully-depleted PSF based on the known velocity-field relation for electrons in high-purity silicon [11]. The smaller PSF values assume that the transverse diffusion coefficient is suppressed at high fields according to the model of [12]. We are investigating alternative measurements of PSF using $\mathrm{x}$-ray cluster size and cosmic ray track profiles to help understand the discrepancy between the VKE results and theory. Initial results from these new techniques show somewhat smaller PSF, in better agreement with the theory.
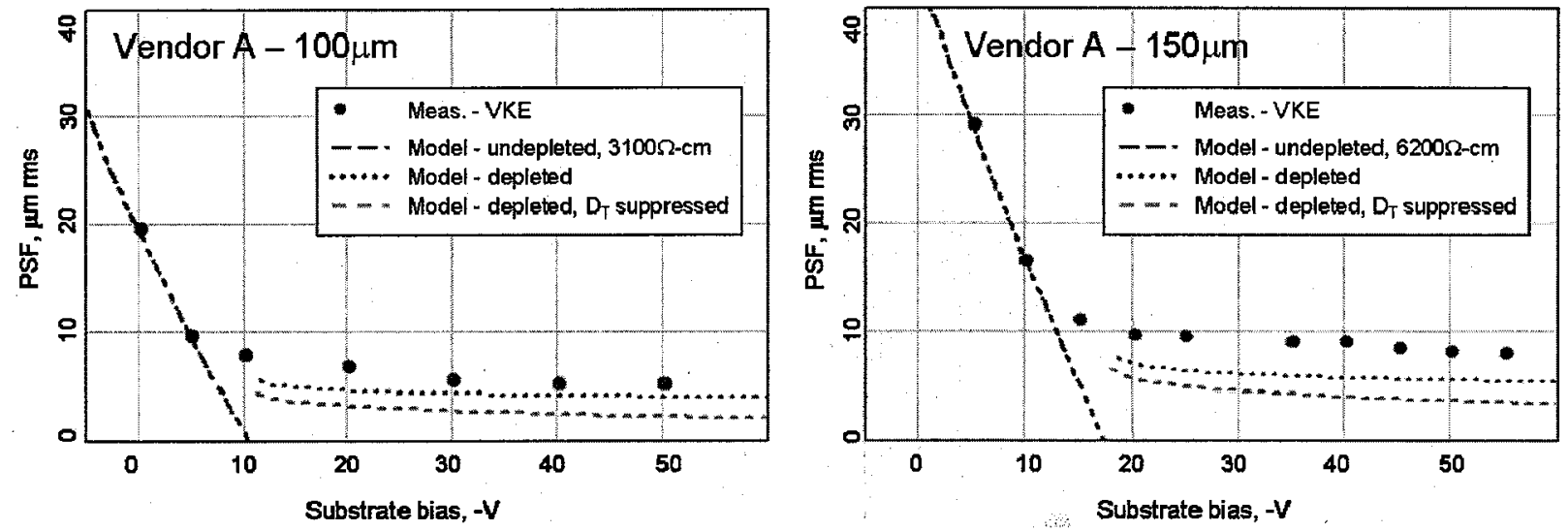

Figure 12: Point spread function measured by the virtual knife edge technique for samples 106-05 (left) and 107-01 (right).

\subsection{Image persistence}

One device from Vendor A was tested for image persistence. A point source of red light was projected onto the surface of the CCD and the intensity and exposure time adjusted to produce about $1 / 3$ of full well in the brightest pixel. The exposures were repeated sixteen times without moving the spot, then an image was taken immediately with the source turned off. As shown in Figure 13, image persistence was less than $0.03 \%$, with the measurement limited by signal to noise.
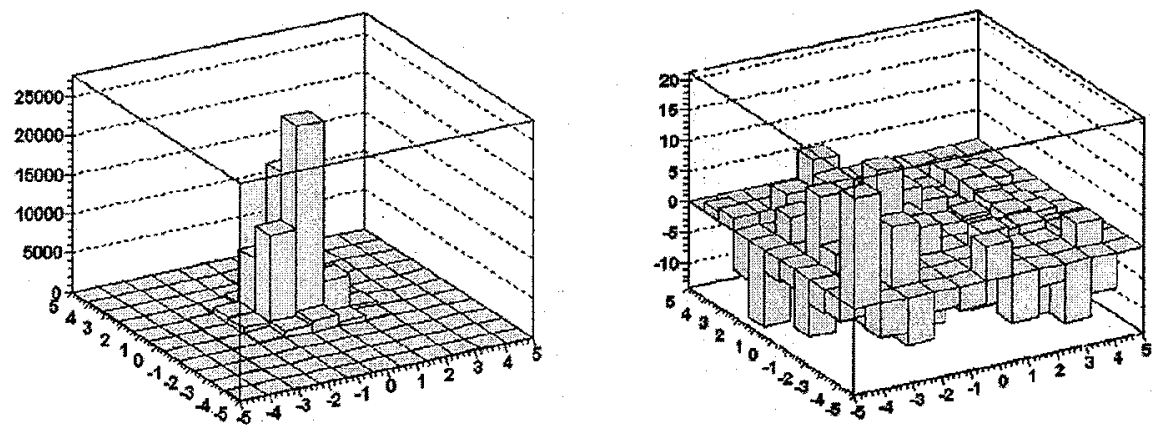

Figure 13: Absence of image persistence in n-channel CCD 106-05. Left: average of 16 exposures of point source. Right: first exposure after source turned off. Persistence is less than $0.03 \%$.

\subsection{Mosaic assembly}

As discussed in Section 2, LSST's fast optics require exceptional flatness of the mosaic focal plane. We experimented with a method similar to that described in [13] to adjust the tilts of dummy sensors on a prototype raft baseplate. The dummy sensors were assembled onto the raft with 3-point spacers whose heights could be adjusted by machining or 
lapping. Each trial assembly was scanned with the confocal displacement sensor, tilts measured, and spacers adjusted to make all surfaces coplanar. Initial tests using this approach resulted in overall flatness of 11 microns peak-to-valley, somewhat less than the LSST requirement. Figure 14 shows the progress of one series of adjustment trial assemblies. Further tests and alternative methods of planarization are being investigated.

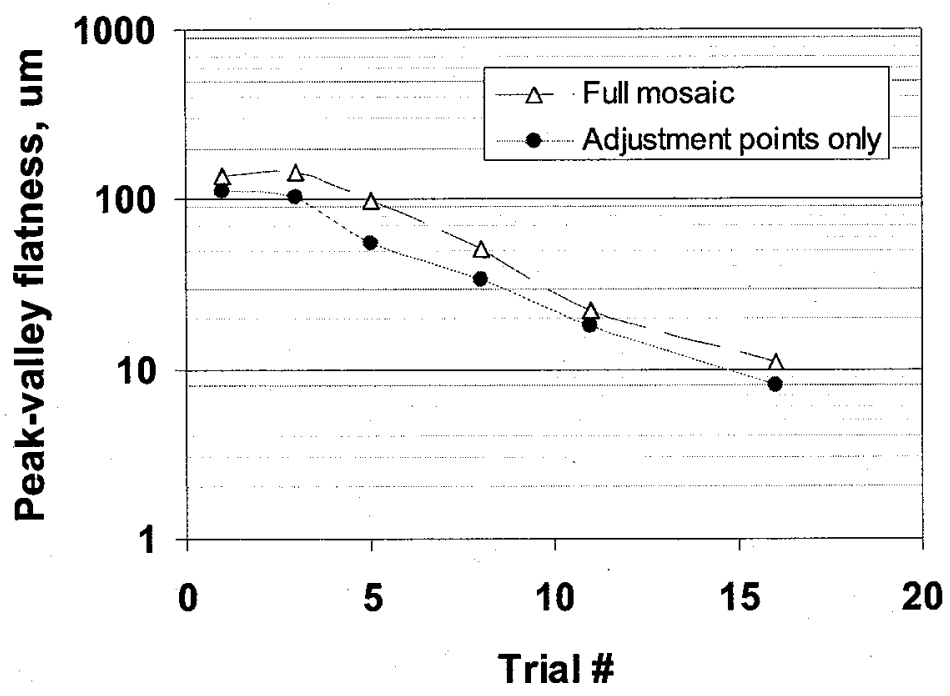

Figure 14: Flatness of dummy sensor mosaic adjusted using machined spacers. Solid points: peak-to-valley variation of height over support points. Triangles: flatness of full mosaic.

\section{SUMMARY AND OUTLOOK}

The LSST sensor study program has been an important step towards development of the advanced sensors needed to achieve the survey's ambitious science goals. Vendors participating in the study program have demonstrated viable approaches to producing devices having extended red response with $100 \mu \mathrm{m}$-thick, fully-depleted silicon; transparent back window contact; low dark current; negligible image persistence; high full well; flat silicon surface; and multiport output for fast readout. A characterization laboratory at BNL with fully automated image acquisition and analysis has verified the performance characteristics of the devices. The collaboration now plans to work with vendors to develop a full prototype meeting all critical LSST specifications that will allow procurement to begin in 2011.

\section{ACKNOWLEDGEMENTS}

This report was prepared as an account of work sponsored by an agency of the United States Government. Neither the United States Government nor any agency thereof, nor any of their employees, nor any of their contractors, subcontractors, or their employees, makes any warranty, express or implied, or assumes any legal liability or responsibility for the accuracy, completeness, or any third party's use or the results of such use of any information, apparatus, product, or process disclosed, or represents that its use would not infringe privately owned rights. Reference herein to any specific commercial product, process, or service by trade name, trademark, manufacturer, or otherwise, does not necessarily constitute or imply its endorsement, recommendation, or favoring by the United States Government or any agency thereof or its contractors or subcontractors. The views and opinions of authors expressed herein do not necessarily state or reflect those of the United States Government or any agency thereof. The publisher by accepting the manuscript for publication acknowledges that the United States Government retains a non-exclusive, paid-up, irrevocable, world-wide license to publish or reproduce the published form of this manuscript, or allow others to do so, for United States Government purposes. This preprint is intended for publication in a journal or proceedings. Since changes may be made before publication, it may not be cited or reproduced without the author's permission. 


\section{REFERENCES}

[1] P. O'Connor et al., Study of silicon thickness optimization for LSST, 15 June 2006, Proceedings Vol. 6276, High Energy, Optical, and Infrared Detectors for Astronomy II, David A. Dorn; Andrew D. Holland, Editors, 62761W

[2] J.C. Geary et al., The LSST Sensor technologies studies, Proc. SPIE, vol. 6276, 627601

[3] Lance M. Simms et al., First use of a HyViSI H4RG for astronomical observations, Proc. SPIE, Vol. 6690, 2007

[4] P.Z. Takacs et al., Detector module and raft assembly metrology concepts, Proc. SPIE, Vol 6273

[5] J.C. Geary, S. Amato, Electronics for the 72-channel SAO Megacam, Optical astronomical instrumentation; S. D'Odorico ed., SPIE 3355, pp. 539-546. 1998; J.C. Geary, Signal processing for the SAO Megacam, in "Further Developments in Scientific Optical Imaging", M.B. Denton ed., Royal Society of Chemistry, Cambridge, 2000, 1823

[6] PCI DV FOX, Engineering Design Team, Inc., www.edt.com

[7] P. Kubanek et al., Remote telescope system, $2^{\text {nd }}$ version, in Gamma-Ray Bursts: 30 Years of Discovery: GammaRay Burst Symposium. AIP Conference Proceedings, Vol. 727, held 8-12 September, 2003 in Santa Fe, New Mexico. Edited by E. E. Fenimore and M. Galassi. Melville, NY: American Institute of Physics, 2004., p.753-756

[8] A. Karcher et al., Measurement of Lateral Charge Diffusion in thick, fully depleted, back-illuminated CCDs, IEEE Trans. Nucl. Sci. 51(5), 2231, Oct. 2004

[9] J. Fairfield et al., J. A.Fairfield et al, "Reduced Charge Diffusion in Thick, Fully Depleted CCDs With Enhanced Red Sensitivity", IEEE Transactions on Nuclear Science, Vol 53, 3877-3881, 2006

[10] K. Rajkanan et al., Sol. St. Electr. 22, 793-795 (1979)

[11] C. Canali, G. Majni, R. Minder, and G. Ottaviani., "Electron and Hole Drift Velocity Measurements in Silicon and Their Empirical Relation to Electric Field and Temperature", IEEE Trans. Electron Dev. Vol. 22, pp.1045-1046, 1975

[12] V. Arora, Drift diffusion and Einstein relation for electrons in silicon subjected to a high electric field, Applied Physics Lett. 80(20), 3763 (May 2002); also see V. Radeka, Charge diffusion PSF in thick over-depleted silicon sensors, presented at $6^{\text {th }}$ International Conference on Scientific Optical Imaging, Cozumel, Mexico, Dec. 2006.

[13] S.M.Faber et al., The DEIMOS spectrograph for the Keck II telescope: integration and testing,Proc. SPIE 4841, p. 1657-1669, March 2003 